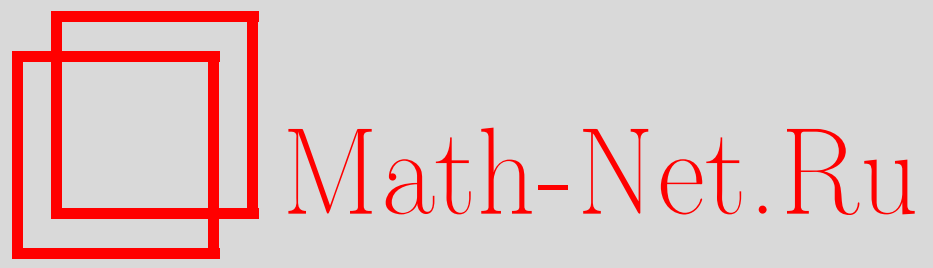

Обцероссийский математический портал

А. А. Космодемьянский, Третья краевая задача для уравнения Пуассона, Матем. заметки, 1998, том 63, выпуск 2, 296-298

DOI: https://doi.org/10.4213/mzm1278

Использование Общероссийского математического портала Math-Net.Ru подразумевает, что вы прочитали и согласны с пользовательским соглашением http://www.mathnet.ru/rus/agreement

Параметры загрузки:

IP: 3.82 .47 .9

26 апреля 2023 г., 17:49:03 


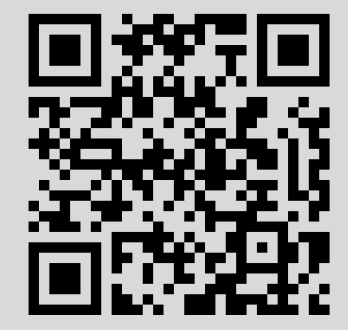




\section{ТРЕТЬЯ КРАЕВАЯ ЗАДАЧА ДЛЯ УРАВНЕНИЯ ПУАССОНА}

\section{А. А. Космодемьянский}

Третья краевая задача состоит в отыскании решения уравнения $\Delta u=-1$ с граничными условиями

$$
u+\beta \frac{\partial u}{\partial n}=0, \quad \beta>0 .
$$

Покажем, как методами [1] получить изопериметрические оценки решения такой задачи в плоской выпуклой области $D$ с достаточно гладкой границей $\Gamma$.

Оценки, использующие принцип максимума, получены в [2], где, в частности, доказана положительность функции $u$. Согласно [2] поставленную задачу будем называть задачей $P(\beta)$.

Пусть $|\Gamma|$ - периметр границы $\Gamma$, а $|D|$ - площадь области $D$. Напомним, что $\partial u / \partial n=u_{n}-$ производная по внешней нормали к границе $\Gamma$.

Теорема 1. Пусть кривизна границы Г удовлетворяет неравенству $K \geqslant K_{\min }>0 u$ пусть и-решение уравнения Пуассона с граничными условиями (1). Тогда

$$
\begin{aligned}
& \max _{\Gamma} u \leqslant \frac{\beta|D|}{|\Gamma|}+\left(\frac{|D|}{2}-\frac{|D|^{2}}{J}\right) \beta^{1 / 2}\left(\int_{\Gamma} \frac{d s}{K \beta+2}\right)^{1 / 2} \\
& \min _{\Gamma} u \geqslant \frac{\beta|D|}{|\Gamma|}-\left(\frac{|D|}{2}-\frac{|D|^{2}}{J}\right) \beta^{1 / 2}\left(\int_{\Gamma} \frac{d s}{K \beta+2}\right)^{1 / 2}
\end{aligned}
$$

əде $J=\int_{\Gamma} \frac{d s}{K}$

ДоКАЗАТЕЛЬСТВо близко к доказательству соответствующей теоремы из [1]. Пусть $G=$ $u_{x x} u_{y y}-u_{x y}^{2}$. Тогда

$$
2 \iint_{D} G d x d y=\int_{\Gamma}\left(K \dot{u}^{2}-2 \dot{u} \dot{u}_{n}+K u_{n}^{2}\right) d s .
$$

Здесь и далее точкой обозначается производная по натуральому параметру вдоль $\Gamma$.

Выразим теперь $u_{n}$ и $\dot{u}_{n}$ из граничных условий (1) и подставим в формулу (4). Получим

$$
2 \iint_{D} G d x d y=\int_{\Gamma}\left(K \dot{u}^{2}+\frac{2}{\beta} \dot{u}^{2}\right)+\frac{K u^{2}}{\beta^{2}} d s .
$$

Левую часть равенства (5) оценим с помощью очевидного неравенства $G \leqslant 1 / 4$. Имеем

$$
2 \iint_{D} G d x d y \leqslant \frac{|D|}{2} .
$$

(C) А.А. КосмодемьянСКий 
Оценим теперь правую часть (5). Интегрируя исходное уравнение по области $D$ и учитывая граничные условия (1), получим

$$
\int_{\Gamma} \frac{\partial u}{\partial n} d s=-\frac{1}{\beta} \int_{\Gamma} u d s=-|D| .
$$

Из равенства (7) выводится оценка

$$
(\beta|D|)^{2}=\left(\int_{\Gamma} u d s\right)^{2}=\left(\int_{\Gamma} \frac{u \sqrt{K}}{\sqrt{K}} d s\right)^{2} \leqslant \int_{\Gamma} K u^{2} d s \cdot J .
$$

Таким образом, из равенства (5) и оценок (6) и (8) получим неравенство

$$
\int_{\Gamma}\left(K \dot{u}^{2}+\frac{2}{\beta} \dot{u}^{2}\right) d s \leqslant \frac{|D|}{2}-\frac{|D|^{2}}{J}
$$

или

$$
\int_{\Gamma}(K \beta+2) \dot{u}^{2} d s \leqslant\left(\frac{|D|}{2}-\frac{|D|^{2}}{J}\right) \beta .
$$

Далее рассуждаем как в работе [1]. Из формулы (7) видно, что на границе $Г$ есть точка $M_{0}$ такая, что $u\left(M_{0}\right)=\beta|D| /|\Gamma|$. Пусть $\Gamma_{0}-$ дуга $\Gamma$, концами которой служат точка $M_{0}$ и точка, в которой функция $u$ достигает своего максимума на $Г$. Тогда

$$
\max _{\Gamma} u-\frac{\beta|D|}{|\Gamma|}=\int_{\Gamma_{0}} \dot{u} d s .
$$

Возведем обе части последнего равенства в квадрат и осуществим оценку, используя неравенство (9):

$$
\begin{aligned}
\left(\max _{\Gamma} u-\frac{\beta|D|}{|\Gamma|}\right)^{2} & =\left(\int_{\Gamma_{0}} \dot{u} d s\right)^{2}=\left(\int_{\Gamma_{0}} \frac{\sqrt{K \beta+2} \dot{u}}{\sqrt{K \beta+2}} d s\right)^{2} \\
& \leqslant \int_{\Gamma}(K \beta+2) \dot{u}^{2} d s \int_{\Gamma} \frac{d s}{K \beta+2} \leqslant\left(\frac{|D|}{2}-\frac{|D|^{2}}{J}\right) \beta \int_{\Gamma} \frac{d s}{K \beta+2}
\end{aligned}
$$

Извлекая корень, получим неравенство (2). Неравенство (3) доказывается аналогично.

При малых $\beta$ неравенства (2) и (3) слишком грубы. В этом случае их естественно заменить другими, точньми по порядкку.

Теорема 2. В условиях теоремы 1 справедливы неравенства

$$
\frac{\beta}{2 K_{\min }} \geqslant \max _{\Gamma} u \geqslant \frac{\beta|D|}{|\Gamma|}, \quad \frac{\beta}{2 K_{\max }} \leqslant \min _{\Gamma} u \leqslant \frac{\beta|D|}{|\Gamma|} .
$$

ДоказАтЕЛЬСтво. Правые части неравенств (10) следуют из формулы (7). Докажем теперь левую часть первого из неравенств. Пусть $u_{0}-$ решение задачи $P(0)$. Рассмотрим функцию $w=$ $u_{0}+\max _{\Gamma} u-u$. Функция $w$ положительна и гармонична в области $D$ и обращается в 0 в той точке границы $\Gamma$, где функция $u$ достигает своего максимума. Следовательно, по лемме о нормальной производной в этой точке $w_{n}<0$. Отсюда немедленно следует

$$
-\frac{\partial u_{0}}{\partial n}>-\frac{\partial u}{\partial n}=\frac{1}{\beta} u_{\max } .
$$

Таким образом,

$$
u_{\max }<\beta \max _{\Gamma}\left|\frac{\partial u_{0}}{\partial n}\right| .
$$


Для завершения доказательства воспользуемся неравенством, полученньм в [3]:

$$
\frac{1}{2 K_{\max }} \leqslant\left|\frac{\partial u_{0}}{\partial n}\right| \leqslant \frac{1}{2 K_{\min }} .
$$

Левая часть второго неравенства в (10) доказывается аналогично. Заметим, что обращение каждого из неравенств $(10)$ в равенство возможно лишш тогда, когда область $D$ - круг. Это утверждение следует из строгого принципа максимума.

Аналогичные оценки получены в [2].

Доказанные теоремы дают возможность проследить за поведением решения третьей краевой задачи при $\beta \rightarrow 0$ и $\beta \rightarrow \infty$.

Пусть $u_{0}$ - решение задачи $P(0)$. Поскольку разность $u-u_{0}$ является гармонической функцией, из принципа максимума и теоремы 2 получаются оценки

$\max _{D}\left|u-u_{0}\right| \leqslant \max _{\Gamma}\left|u-u_{0}\right|=\max _{\Gamma} u \leqslant \frac{\beta}{2 K_{\min }}, \quad \min _{D}\left|u-u_{0}\right| \geqslant \min _{\Gamma}\left|u-u_{0}\right|=\min _{\Gamma} u \geqslant \frac{\beta}{2 K_{\max }}$.

При больших $\beta$ неравенство (2) принимает вид

$$
\max _{\Gamma} u \leqslant \frac{\beta|D|}{|\Gamma|}+\left(\frac{|D|}{2}-\frac{|D|^{2}}{J}\right)^{1 / 2} J^{1 / 2},
$$

поэтому оценки (2), (3) теоремы 1 в силу условий (1) могут быть представлены в следующей форме:

$$
\max _{\Gamma}\left|\frac{\partial u}{\partial n}\right| \leqslant \frac{|D|}{|\Gamma|}+\left(\frac{|D|}{2}-\frac{|D|^{2}}{J}\right)^{1 / 2} \frac{J^{1 / 2}}{\beta}, \quad \min _{\Gamma}\left|\frac{\partial u}{\partial n}\right| \geqslant \frac{|D|}{|\Gamma|}-\left(\frac{|D|}{2}-\frac{|D|^{2}}{J}\right)^{1 / 2} \frac{J^{1 / 2}}{\beta} .
$$

Пусть $v_{\beta}=u-\beta|D| /|\Gamma|$, а $u_{\infty}$ - решение исходного уравнения с граничными условиями

такое, что

$$
\frac{\partial u_{\infty}}{\partial n}=-\frac{|D|}{|\Gamma|}
$$

Из формулы (7) следует, что

$$
\int_{\Gamma} u_{\infty} d s=0
$$

$$
\int_{\Gamma} v_{\beta} d s=0
$$

а из формул (11) - неравенства

$$
\frac{|D|}{|\Gamma|}-\frac{1}{\beta}\left(\frac{|D|}{2}-\frac{|D|^{2}}{J}\right)^{1 / 2} J^{1 / 2} \leqslant \frac{\partial v_{\beta}}{\partial n} \leqslant \frac{1}{\beta}\left(\frac{|D|}{2}-\frac{|D|^{2}}{J}\right)^{1 / 2} J^{1 / 2}+\frac{|D|}{|\Gamma|} .
$$

Поэтому можно оценить нормальную производную гармонической функции $v=u_{\infty}-v_{\beta}$ :

$$
-\frac{1}{\beta}\left(\frac{|D|}{2}-\frac{|D|^{2}}{J}\right)^{1 / 2} J^{1 / 2} \leqslant \frac{\partial v}{\partial n} \leqslant \frac{1}{\beta}\left(\frac{|D|}{2}-\frac{|D|^{2}}{J}\right)^{1 / 2} J^{1 / 2} .
$$

Кроме того,

$$
\int_{\Gamma} v d s=0
$$

Поэтому при $\beta \rightarrow \infty$ функция $v$ стремится к 0 , а значит, функция $v_{\beta}-$ к функции $u_{\infty}$.

\section{СПИСОК ЦИТИРОВАННОЙ ЛИТЕРАТУРЫ}

1. Космодемьянский А. А. // Матем. заметки. 1991. Т. 50. № 4. С. 48-53. 2. Keady G., McNabb A. // IMA J. Appl. Math. 1993. V. 50. P. 205-224. 3. Космодемьянский А. А. // Матем. заметки. 1987. Т. 42. № 4. С. 537-542. 